\title{
A COMBINED PHOTON-HADRON HODOSCOPE CALORIMETER
}

$$
\text { IHEP }^{1}-\text { IISN }^{2}-\text { LAPP }^{3} \text { Collaboration }
$$

F.G. Binon ${ }^{2 *}$, C. Bricman ${ }^{2 *}$, P. Duteil ${ }^{4}$, M. Gouanère ${ }^{3}$, V.A. Kachanov ${ }^{2}$, D.B. Kakauridze ${ }^{1+}$, G.V. Khaustov ${ }^{2}$, J.P. Lagnaux ${ }^{2 *}$, Th. Mouthuy ${ }^{2}$, J.P. Peigneux ${ }^{3}$, A. Possoz ${ }^{4}$ Yu.D. Prokoshkin', V.I. Rykalin ${ }^{2}$, P.M. Shagin ${ }^{1}$, D. Sillou ${ }^{3}$, A.V. Singovsky ${ }^{2}$, J.P. Stroot $^{2^{\star}}$ and V.P. Sugonyaev ${ }^{1}$

(Joint CERN-IHEP experiment)

\section{ABSTRACT}

The energy and space resolution and other characteristics of a combined detector permitting simultaneous detection of a large number of photons and hadrons has been studied in a $200 \mathrm{GeV} / \mathrm{c}$ hadron beam at the CERN SPS. The detector consists of the GAMS-4000 spectrometer (4096 lead-glass cel1s) and a modular hadron calorimeter MHC-200 located behind it (240 total-absorption sandwich counters). A new method for adding the signals from the two calorimeters, which takes into account the difference in the developement of hadron showers in photon and hadron calorimeters, has been developed. It permits substantial improvement of the energy resolution of the combined detector. The hadron coordinates are defined with a precision of several millimetres and the energy resolution is typical for steel-scintillator sandwiches with a wavelength-shifter read-out. The effect of the gap between the photon and the hadron calorimeters has also been studied.

(Submitted to Nuclear Instruments and Methods)

+) Deceased

1) Institute for High Energy Physics, Serpukhov, USSR.

2) Institut Interuniversitaire des Sciences Nucléaires, Belgium.

3) Laboratoire d'Annecy de Physiques des Particules, France.

4) CERN, Geneva, Switzerland.

*) Mailing address: CERN, EP Division, CH-1211 Geneva 23, Switzerland 
1. INTRODUCTION

The characteristics of a combined detector comprising the GAMS 4000 spectrometer [1] and the modular hadron calorimeter MHC-200 [2] 1ocated in the 48 beam of the CERN SPS have been studied. GAMS-4000 and MHC-200 have already been described as individual detectors [1-4]. A study of their joint operation for detecting hadrons is important since different physical processes are used in these two calorimeters for shower detection (via Cerenkov radiation in GAMS and via ionization losses in MHC). The way to add hadron signals in both detectors is not clear a priori.

High-granularity calorimeters which make it possible to measure simultaneously energy and coordinates of a large number of photons and hadrons, are increasingly becoming basic elements of experimental setups at the highest energies [5]. There is a need to develop detectors made to record both photons (electrons) and hadrons simultaneously [6].

In this paper are reported measurements in a hadron beam of the coordinate and energy resolutions of a combined detector and of the dependence of its characteristics on the distance between the photon and the hadron calorimeters. A new method to improve the energy resolution and to suppress non-linearities when the signals are sumed has been developed taking into account the difference in hadron shower development in the photon and hadron calorimeters.

\section{EXPERIMENTAL APPARATUS}

The measurements have been performed in a $200 \mathrm{GeV} / \mathrm{c}$ beam of hadrons comprising roughly equal proportion of $\pi^{+}$and protons. Contamination from other particles was smal1. The beam was deflected by a magnet in the horizontal plane in order to scan the detectors. The coordinates of the beam particles were determined by a scintillation counter, 2 mm wide and $30 \mathrm{~mm}$ high, which was located in front of GAMS -4000 . 
The overall layout of the combined detector is shown in fig 1 . The GAMS -4000 spectrometer is a matrix of $64 \times 64$ counters with radiators made of transparent lead glass with transverse $38 \times 38 \mathrm{~mm}^{2}$ dimensions (for details see paper [3] where a similar detector of smaller size is described). The length of the lead-glass cells ( $45 \mathrm{~cm}, 16$ radiation lengths) is sufficient for practically assuring total absorption of electromagnetic showers with an energy up to several hundred Gev. When hadrons are passing through GAMS, a significant fraction of them $(\approx 70 \%)$ also start to shower in the lead-glass cells. However, GAMS serves only as a thin active converter for hadrons (about one nuclear absorption length). The hadron showers emerging from GAMS are entirely absorbed in MHC-200.

MHC-200 is a matrix of $13 \times 13$ modules with $20 \times 20 \mathrm{~cm}^{2}$ transverse dimensions. The 24 central modules, which surround a $20 \times 20 \mathrm{~cm}^{2}$ hole for the beam, comprise four sandwich-type total absorption counters measuring $10 \times 10 \mathrm{~cm}^{2}$ each. The other 144 modules make up a single $20 \times 20 \mathrm{~cm}^{2}$ counter. Each total absorption counter consists of thirty-six $2.5 \mathrm{~cm}$ thick steel plates sandwiched with $5 \mathrm{~mm}$ scintillator sheets ( 5 nuclear absorption lengths for pions and 7 nuclear absorption lengths for protons). The scintillator light is collected by a flat light guide with a wavelength shifter. The full calorimeter aperture covers $6 \mathrm{~m}^{2}$. More details on MHC-200 are given in ref. [2].

The electronics of the data acquisition system used in the combined detector have already been described elsewhere [7].

The minimum distance between the rear end of GAMS-4000 lead-glass cells and the front end of MHC-200 modules is determined by the design of GAMS-4000: it is $50 \mathrm{~cm}$. This distance may be increased up to $2 \mathrm{~m}$ by moving the 50-ton MHC-200 on rails.

The calorimeters were calibrated in muon, electron and hadron beams. The calibration procedures are described elsewhere $[2,3]$.

The study of the characteristics of the combined detector has been performed with the hadron beam entering the central region of a MHC-200 counter $\left(20 \times 20 \mathrm{~cm}^{2}\right)$, after traversing GAMS -4000 . 


\section{HADRON ENERGY MEASUREMENT}

Figure 2 represents a two-dimensional plot of the energy deposited in both calorimeters by $200 \mathrm{GeV}$ hadrons. The total signal of the combined detector may be expressed as

$$
A_{\text {tot }}=a^{\circ}{ }_{G} A_{G}+a^{\circ}{ }_{H} A_{H} \text {. }
$$

The normalization coefficients $a^{\circ}{ }_{G}$ and $a^{\circ}$ have been obtained by minimizing the energy resolution of the combined detector for the events in the range indicated on fig. 2 by the dashed lines.

The spectra of the hadron signals in GAMS-4000 and in MHC-200 are shown in fig. 3 after normalization with these coefficients. The narrow peak in the region of very small amplitudes in GAMS corresponds to hadrons which traverse GAMS without interacting (30\%).

Figure 4a shows the $A_{\text {tot }}$ spectrum obtained according to relation (1). The energy resolution is $\sigma_{E} / E=9.3 \%$, i.e. 1.4 times larger than that of MHC-200 alone [2].

Figure 2 shows that non-1inearity is specially important when the hadrons release most of their energy in GAMS, i.e. when $a=a^{\circ}{ }_{G}{ }_{G} / A_{\text {tot }} \approx 1$. (see also [8]). In this case the mean value of $A_{\text {tot }}$ is larger than the beam energy. The distribution of $A_{\text {tot }}$ for these events is rather asymetrical (fig. 4c) (the fraction of such events is small, see fig. 3 ).

Attempts to correct the observed spectra with a-dependent normalization coefficients $a^{\circ}$ remove the systematic shift, but spectra remain asymmetrical and the energy resolution is not improved.

The largest signals in GAMS-4000 $(\alpha \approx 1)$ come from hadron showers which develop through $\pi^{\circ}$ emission and have an important electromagnetic component, which is effectively absorbed in the photon calorimeter [9]. In planar hadron calorimeters this effect is corrected according to the longitudinal development of the shower $[9,10]$. 
In the present work another method of correction is used. It is based on an analysis of the transverse shape of the shower, observing that hadron showers which give a large energy contribution in the electromagnetic calorimeter are narrower [4]. Fig. 5 gives the distribution of the shower half-width (square root of dispersion) in both GAMS-4000 and MHC-200 as a function of $A_{\text {tot }}$. The correlation is clear: the narrower are showers, the larger are total amplitudes.

As $\alpha$ increases this correlation becomes stronger in GAMS and it becomes weaker in MHC. It is then natural to try the following correction for the total amplitude:

$$
\begin{aligned}
& A_{\text {tot }}^{c}=a_{G} A_{G}^{c}+a_{H} A_{H}^{c} \\
& A_{G, H}^{c}=A_{G, H}\left(1+b_{G, H} D_{G, H}\right)
\end{aligned}
$$

where $D_{G, H}$ are the hadron shower dispersions in GAMS and MHC, respectively. $b_{G, H}$ depend only slightly on energy [11].

Resulting spectra are shown on fig. 4b. The energy resolution of the combined detector is now improved to $\sigma_{E} / E=7 \%$, in agreement with the MHC-200 data [2]. The spectrum for $\alpha>0.8$ is given on $\mathrm{fig}$. 4c. It is symmetrical after correction. In the case of hadrons that do not interact in GAMS $(\alpha \approx 0)$ the resolution after correction is improved by $5 \%$ only.

\section{HADRON COORDINATE MEASUREMENTS}

The coordinates of the point where a hadron enters the cellular calorimeter are determined according to the values of the signals in its cells $[5,7]$. This method is applied to both photon and hadron calorimeters $[4,12,13]$.

The hadron coordinates in GAMS-4000 and in MHC-200 were determined through the center of gravity of the transverse projections of the shower with subsequent correction for the systematic displacement linked to the 
exponential shape of the shower [12,14] (see also [21). The position of the center of gravity of the shower in both calorimeters was determined using the signals from $3 \times 3$ cells centered on the cell which delivers the largest amplitude. The use of a larger number of cells does not improve the coordinate resolution in either GAMS (Fig.6) or MHC [2].

Figure 7 shows how the spatial resolution of GAMS -4000 and of MHC-200 depend on the value of $\alpha$, the relative energy released by hadrons in GAMS. With the increase of $\alpha$, the coordinate resolution of MHC worsens considerably while in GAMS, on the contrary, it improves and for $\alpha>0.6$ it becomes comparable with the precision of measurements of $\gamma$-quanta coordinates $(\approx 2 \mathrm{~mm})$. When the hadron point of impact shifts from the centre of a GAMS cell to its edge, the coordinate accuracy somewhat improves, as it is the case for $Y$ showers (Fig. 6). For very small $\alpha$ ( $\alpha<0.05,40 \%$ of the cases), the precision with which the coordinates are measured in GAMS is defined by the cell dimension $\left(\sigma_{x} \approx 1.5 \mathrm{~cm}\right)$.

From Fig. 7 it can be seen that the hadron coordinates in the combined detector for $\alpha>0.05$ (60\% of the events) must be determined using the photon calorimeter only since additional information from MHC does not improve the coordinate accuracy. At lower $\alpha$, the information from both calorimeters must be taken into account. In this case, the hadron coordinates may be determined with a precision varying between 9 mm and $4 \mathrm{~mm}$ depending whether the hadron enters the cell near its center or near its edge, respectively [2].

\section{INFLUENCE OF THE DISTANCE BETWEEN THE PHOTON AND HADRON CALORIMETERS} ON THE DETECTOR CHARACTERISTICS

A gap of $50 \mathrm{~cm}$ between the calorimeters, as was the case for the measurements reported above, does not impair the energy resolution. The transverse leak of the hadron shower in both devices are insignificant. However, when the gap length is increased, this effect may become noticeable. Furthermore, at large gaps the separation in the hadron calorimeter of the showers originating in GAMS is more difficult. To clarify how critical the size of the gap is for the characteristics of the combined detector, measurements were carried out where the distance $l$ varied from the minimum $(50 \mathrm{~cm})$ to $170 \mathrm{~cm}$. 
The results given in fig. 8 show that the width of the shower increases linearly with $\ell$. Although at $\ell=50 \mathrm{~cm}$ the shower is twice as wide as when GAMS is not in front of MHC, it still remains comparable with the size of the MHC module. The shower loss outside the group of $3 \times 3$ modules is at most 7\% (3\% in MHC-200 alone).

As may be seen from Fig. 8 , the increase in the transverse dimensions of the hadron shower already starts in the GAMS radiators, and therefore the reduction of $\ell$, for example, by half (which would, from a technical point of view, be quite complicated in the case of GAMS) reduces the shower width by only $15 \%$ in total. The possibility provided by the combined detector to separate adjacent hadron showers in practice does not depend on the gap up to $\approx 1 \mathrm{~m}$.

The increase of the shower width with increasing gap is also illustrated in Fig. 9, which shows the fraction of energy released in the central module at various $\alpha$ and the part of shower energy escaping the $3 \times 3$ module assembly.

The value of $A_{\text {tot }}$ and the energy resolution of the combined detector virtually do not change when $\ell$ is increased, with the exception of the largest distances $(\ell>1.5 \mathrm{~m})$, when MHC does not collect the full hadron shower emerging from GAMS.

When GAMS-4000 and MHC-200 are operating jointly, an important question is the effect of the backscattering from the hadron calorimeter (albedo). Although this leakage of energy is small (<2\% [15]), it might be expected that the fragments of the hadron shower entering GAMS as a result of the backscattering from the hadron calorimeter are erroneously identified as photons by the reconstruction program. Fig. 10 shows the distribution of the triggered GAMS -4000 cells at minimum and maximum values of $\ell$. It may be seen that these distributions are virtually indistinguishable and that the mean quantity of triggered GAMS cells is the same in both cases. Consequently, the effect of the albedo on GAMS operation is unobservable. 
The half-width of the hadron shower in GAMS-4000 is small. It varies from 12 to $25 \mathrm{~mm}$ in the range $0.1<\alpha<1$, and the mean number of triggered cells at $\alpha>0.02$ is $\approx 9$. There is little probability that the program of photon shower reconstruction [16] would mistake a wide angle fluctuation of a hadron shower as a low energy $\gamma$-quantum. For example, at a distance from the shower axis larger than $3 \mathrm{~cm}$ less than $1 \%$ of hadron showers appear to contain a fake photon with an energy $E_{\gamma}>1.5 \mathrm{GeV}$. GAMS-4000 makes it possible to separate the electromagnetic showers reliably from a background of hadron showers at distances between their axes of 5 to $10 \mathrm{~cm}$ (depending on the values of $\alpha$ and $E_{\gamma}$ ). This characteristics of GAMS, as photon and hadron detector, was used in a stud: of $x$-particle production near threshold [17]. It made it possible to separate the electromagnetic showers from the decay $x \rightarrow J / \psi+\gamma, J / \psi \rightarrow e^{+}+e^{-}$ against an intense hadron background. The use of a combined detector as the one described, which reliably distinguishes photons and hadrons, simplifies the solution of this type of experimental problem substantially.

\section{CONCLUSION}

Combined detectors in which hodoscope spectrometers of the GAMS type are used as photon calorimeters and in which cellular sandwiches are used as hadron calorimeters are shown to be very promising. Such detectors which preserve the capability of Cerenkov spectrometers to record photons and electrons, also ensure the reconstruction of hadron coordinates and energy with high accuracy. The characteristics of the combined detector proved to be better for recording hadrons than that of a single hadron calorimeter owing to the use of the additional information obtained from the high granularity photon calorimeter.

The energy resolution of the combined detector is similar to that of the hadron calorimeter alone. A correction compensating for the effect of the electromagnetic component fluctuations in the hadron showers makes it possible to eliminate non-1inear effects, so that the energy resolution of the detector improves by $30 \%$ at $200 \mathrm{GeV}$. The coordinates of the hadrons releasing a noticeable amount of energy in GAMS may be measured with an accuracy of 2 to $3 \mathrm{~mm}$. In the worst cases (when the hadron enters the center of a hadron calorimeter cell and the energy release in GAMS is small $(\alpha \approx 0))$, the coordinate resolution of the combined detector is still better than $9 \mathrm{~mm}$. The detector resolution improves with energy as $1 / \sqrt{ } \mathrm{E}$ [5]. 
The presence of a small gap between the calorimeters does not worsen the characteristics of the combined detector. Deterioration is only observable when the gap is larger than $1 \mathrm{~m}$. The backscattering from the hadron calorimeter in GAMS (albedo) is negligible.

The photon calorimeter, by absorbing part of the hadron shower, improves the accuracy of the measurements in the small "hot spots" in the hadron calorimeter, in the region of wavelength-shifter light guide [2].

The authors are grateful to the IHEP and CERN Directorates for their support to the GAMS programme within which this study has been carried out. 


\section{REFERENCES}

[1] F. Binon et al., Preprint IHEP 78-133, Serpukhov (1978).

D. Alde et a1., Nucl. Phys. B269 (1986) 485.

[2] F. Binon, et al., Nucl. Instr. Meth. A256 (1987) 444 .

[3] F. Binon et al., Nuc1. Instr. Meth. A248 (1986) 86 (including further references).

[4] V.A. Davydov et a1., Nuc1. Instr. Meth. 145 (1977) 267.

[5] Yu.D. Prokoshkin, Proceedings of the Second ICFA Workshop on Possibilities and Limitations of Accelerators and Detectors, Les Diablerets, Switzerland, ed. by U. Amaldi (CERN, Geneva (1979)), p. 405; IHEP, 1175, Serpukhov (1985).

[6] G. Gidal et al., Preprint LBL-91, Univ. Calif., Berkeley (1985).

[7] D.Alde et a1., Nucl. Instr. Meth. A240 (1985) 343.

[8] J.A. Appel et a1., Preprint FNAL FN-405, Batavia (1984).

[9] H. Abramowicz et a1., Nuc1. Instr. Meth. 180 (1981) 429.

[10] M. De Vincenzi et a1., Preprint CERN 85-126 (1985).

[11] D. Alde et a1., Preprint IHEP 87-112, Serpurkov (1987); next paper, in this issue.

[12] F. Binon et a1., Nuc1. Instr. Meth. 174 (1980) 369.

[13] F. Binon et al., Nuc1. Instr. Meth. 206 (1983) 373.

[14] S.V. Donskov et a1., Prib. Tech. Exp. 4 (1977) 49.

[15] S. Iwata, Preprint DPNU 13-80, Nagoya Univ. (1980).

[16] A.V. Kulik et al., Preprint IHEP 85-17, Serpukhov (1985).

[17] F. Binon et al., Yad. Fyz. 39 (1984) 640; Nuc1. Phys. B239 (1984) 311. 


\section{FIGURE CAPTIONS}

Fig. 1 Overall view of the combined detector consisting of the hodoscope multiphoton spectrometer GAMS -4000 and the modular hadron calorimeter MHC-200 (experiment NA12 at the CERN SPS).

Fig. 2 Two-dimensional distribution of amplitudes in GAMS-4000 (A ${ }_{G}$ ) and in $M H C-200\left(A_{H}\right)$ (the scales are given in units corresponding to the mean values of the muon signals, $\bar{A}_{\mu}=1$ ). The dashed lines limit the region over which the energy resolution was minimized in a linear approximation (1) (see text).

Fig. 3 Amplitude spectra in GAMS-4000 and MHC-200 for $200 \mathrm{GeV}$ hadrons.

Fig. 4 a) Summed signal $A_{\text {tot }}$, defined by formula(1), of the combined detector;

b) $A_{\text {tot }}^{c}$, defined by formulae (2), (3);

c) A tot defined by formula (1), before correction (dashed histogram) and $A_{\text {tot }}^{c}(3)$ after correction (solid histogram). $\alpha>0.8$

Fig. 5 Two-dimensional distributions of $A_{\text {tot }}$ (1) versus the half-width of the hadron shower in the photon $\left(V D_{G}\right)$ and hadron $\left(V D_{H}\right)$ calorimeters. $D=D_{x}+D_{y}$ is the dispersion of the shower in a plane perpendicular to $i$ ts axis. Here $D_{x}=\sum x_{i}^{2} A_{i} / \Sigma A_{i}$, $x_{i}$ being the distance between the $i-t h$ cell and the shower axis. $0.6<\alpha<0.8$ for GAYS -4000 and $0.2<\alpha<0.4$ for MHC-200. The correlations between $A_{\text {tot }}$ and $V D$ at other values of a are similar.

Fig. 6 Difference between the real hadron coordinate and the coordinates determined from the shower measured in GAYS-4000:

a) $0.6<\alpha<0.8$. The solid histogram shows the distribution of coordinates determined from the signals delivered by $3 \times 3$ cells while the dashed histogram is the corresponding one for $7 \times 7$ cells. b),c) $\alpha>0.05, x_{G}=0$ and $x_{G}=18$ mm correspond to hadrons entering in the centre of a GAMS cell or near its edge, respectively. 
FIGURE CAPTIONS (Cont'd)

Fig. 7 Reconstruction accuracy of $200 \mathrm{GeV}$ hadron coordinates in GAMS -4000 and MHC-200 as a function of $a$. For GAMS the dark points and the solid line correspond to the region near the cell edge, the light points and the dashed line correspond to the centre of the cell.

Fig. 8 a) Change in the half-width of the hadron shower in the MHC-200 calorimeter with the increase of the distance between it and GAMS-4000 (the energy release in GAMS is larger than $2 \bar{A}_{\mu}$, which corresponds to $a>0.01$ ).

b) Same dependence at various values of $\alpha$. The horizontal arrow indicates the half-width of the hadron shower in MHC-200 without the photon calorimeter in front of it. The vertical arrow indicates the position of the middle of the lead-glass GAMS radiators.

Fig. 9 a) Ratio of energy released in a $20 \times 20 \mathrm{~cm}^{2}$ MHC-200 counter, $A_{1}$, to the total energy, $A_{H}$, as a function of the gap between the calorimeters. The hadron passes through the center of the counter.

b) Fraction of the energy released in $\mathrm{MHC}-200$ outside the central group of $3 \times 3$ modules.

Fig. 10 Distribution of triggered cells in GAMS-4000 when its distance to MHC -200 is $\ell=50 \mathrm{~cm}$ and $\ell=140 \mathrm{~cm}$. The number of triggered GAMS cells is proportional to the area of the corresponding square. The cell numbers are indicated along the $x$ and $y$ axes. 


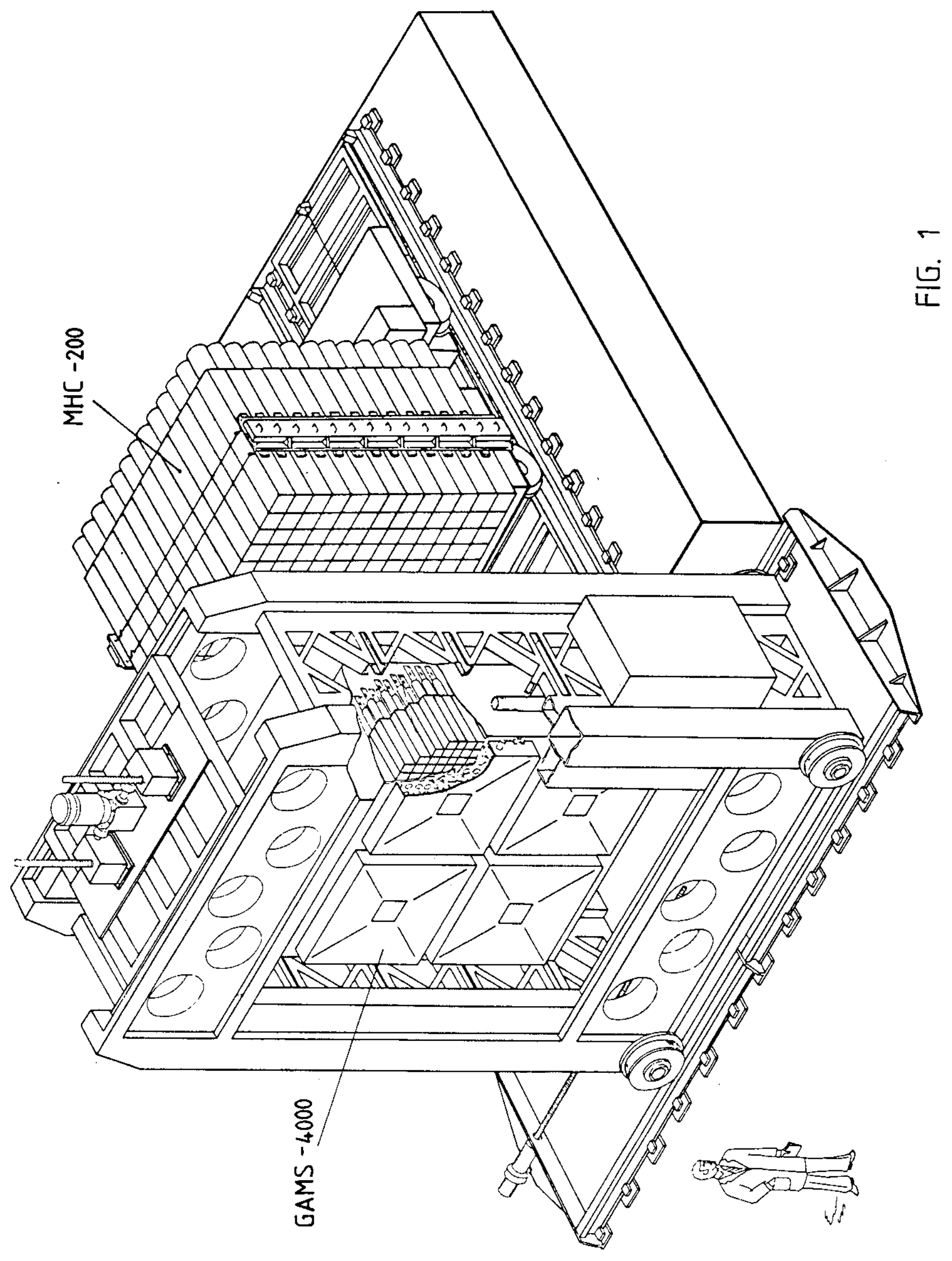




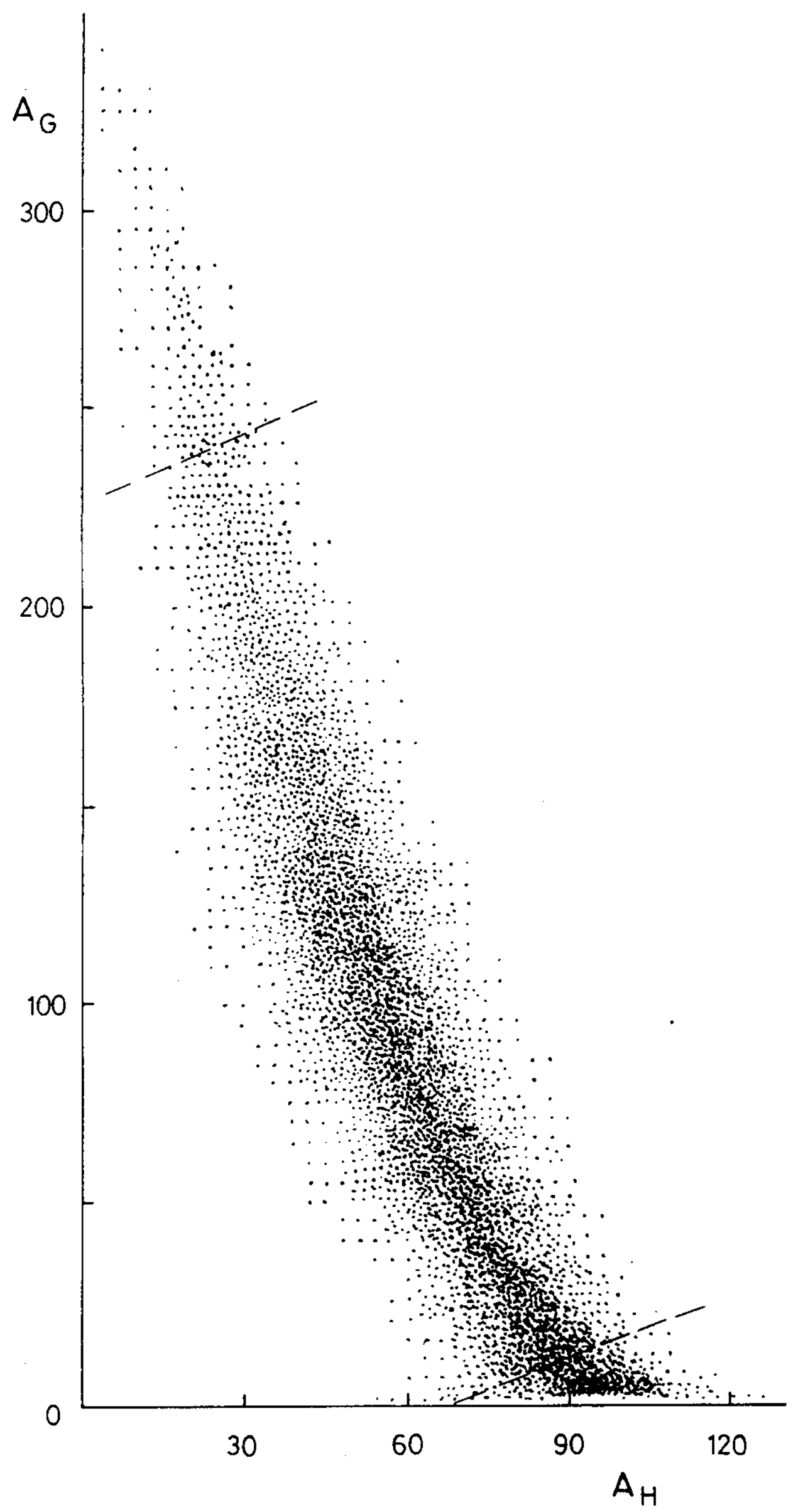

FIG.2 


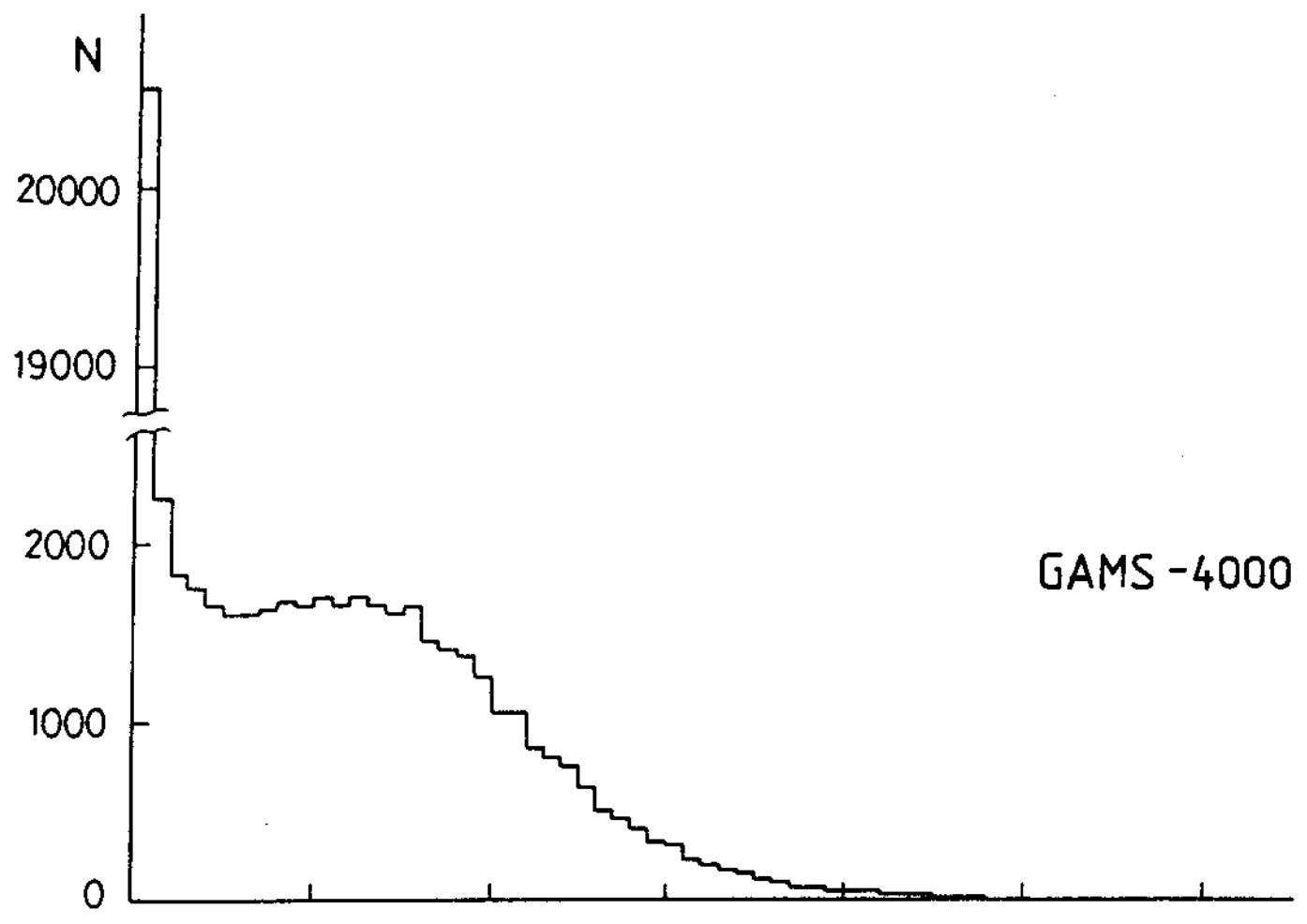

$A_{G}[\mathrm{GeV}]$

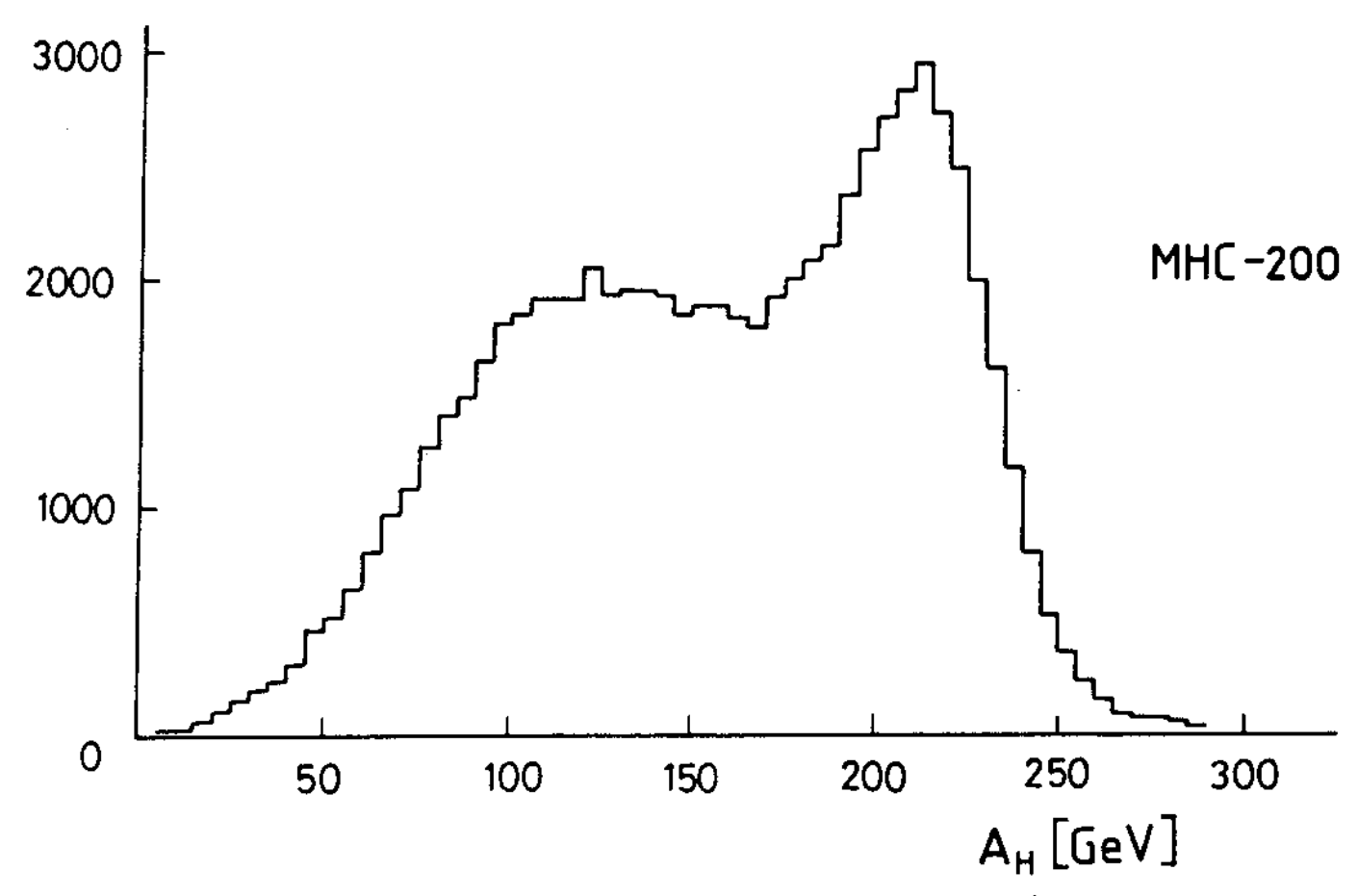

FIG. 3 


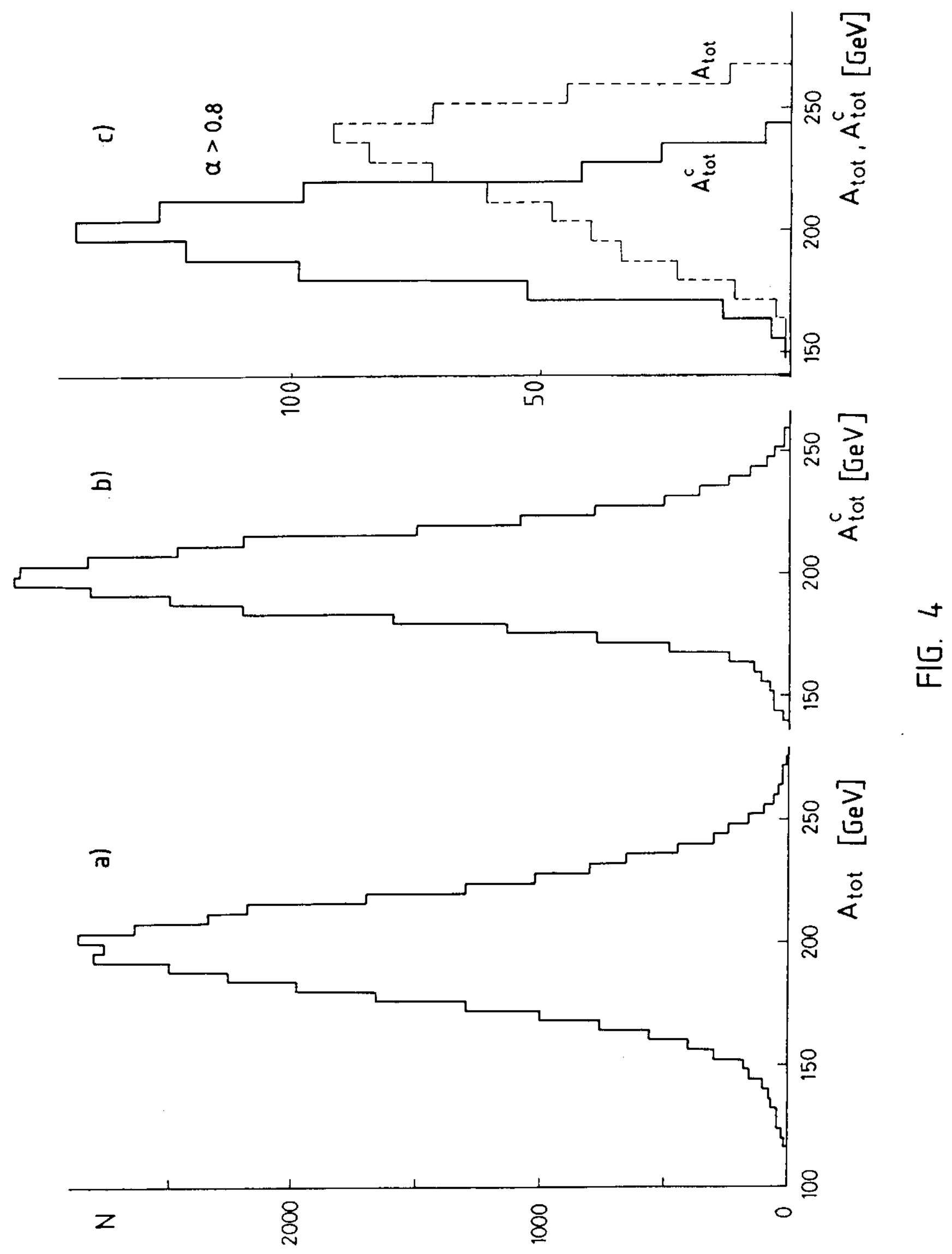



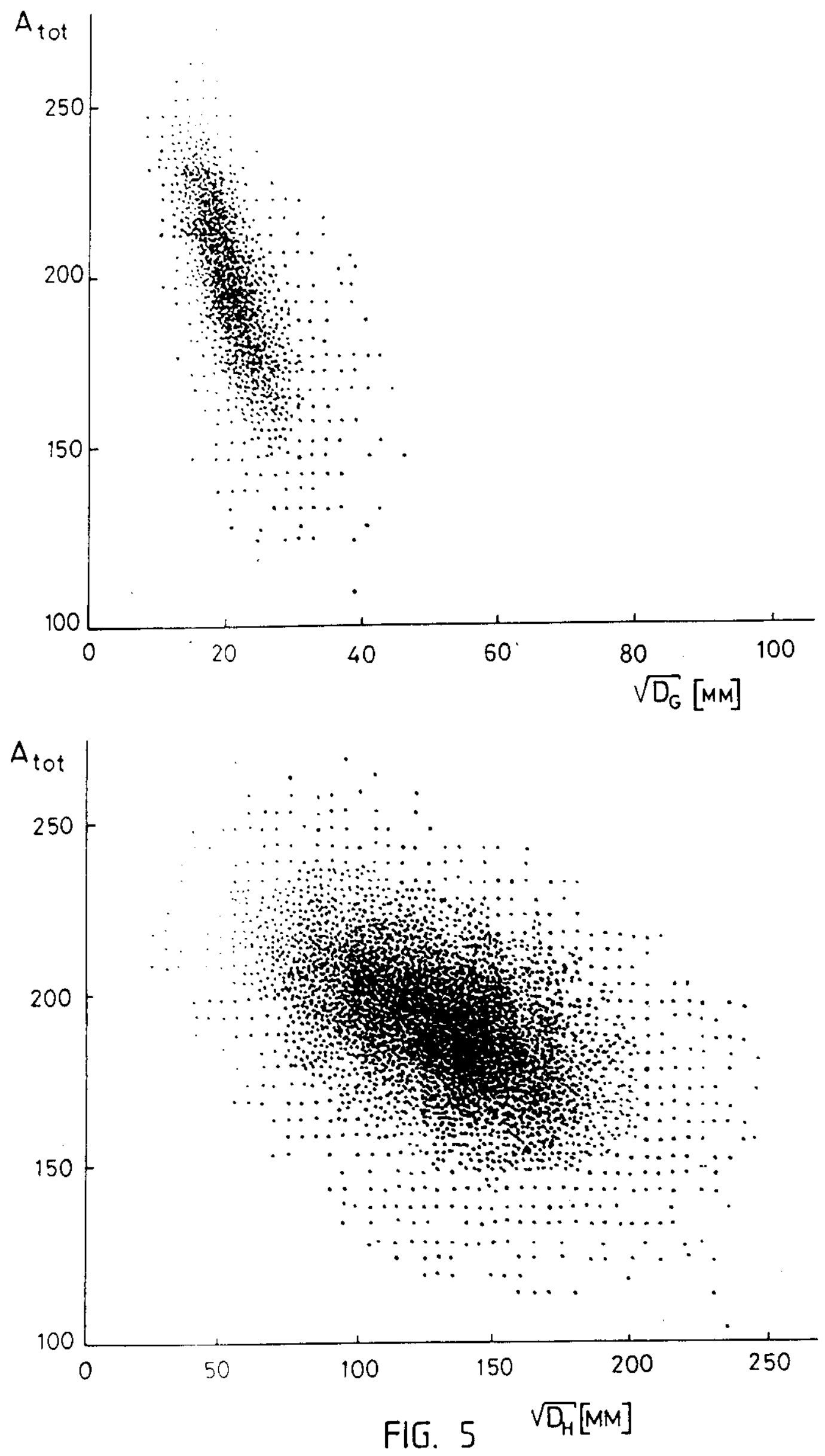

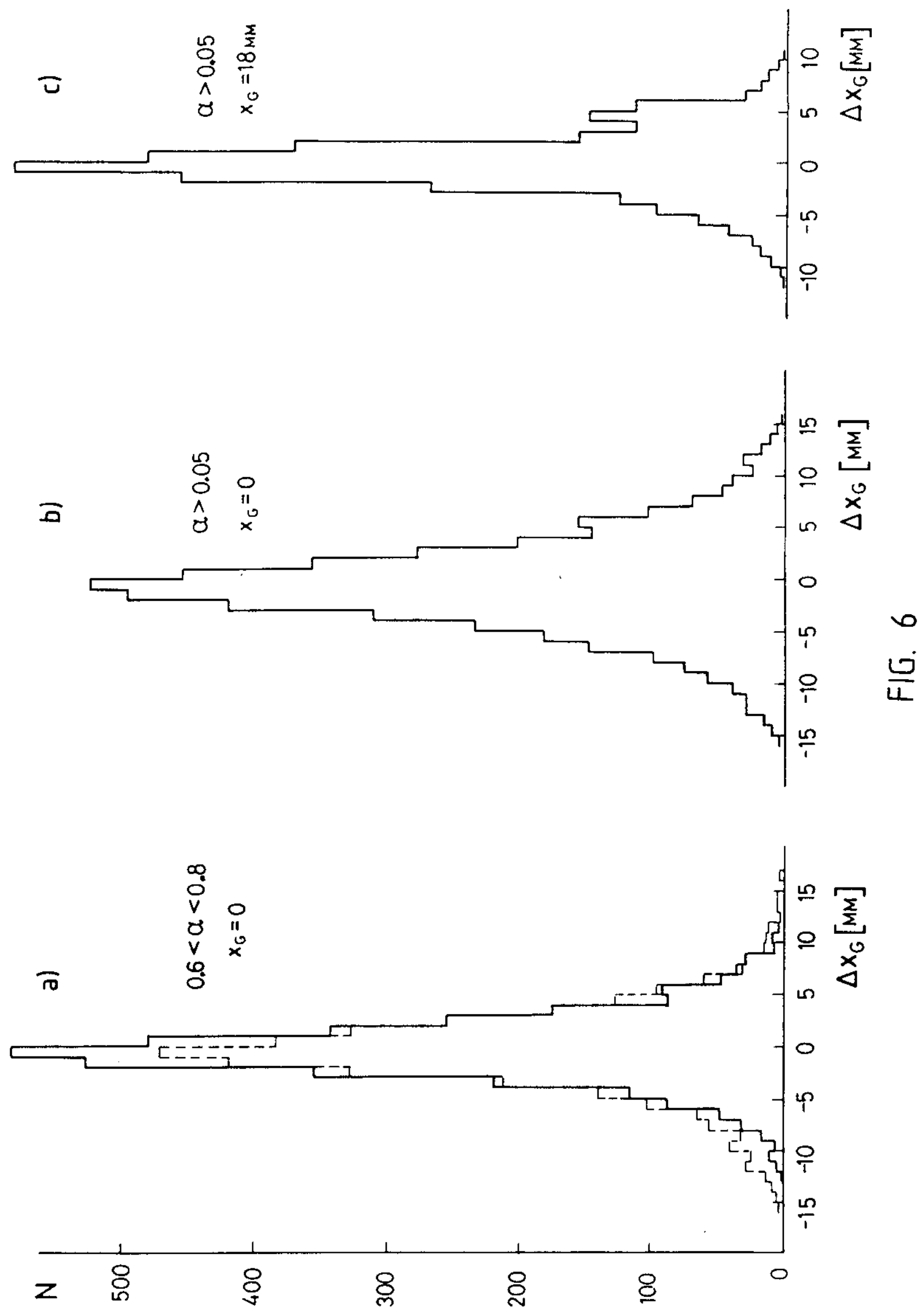


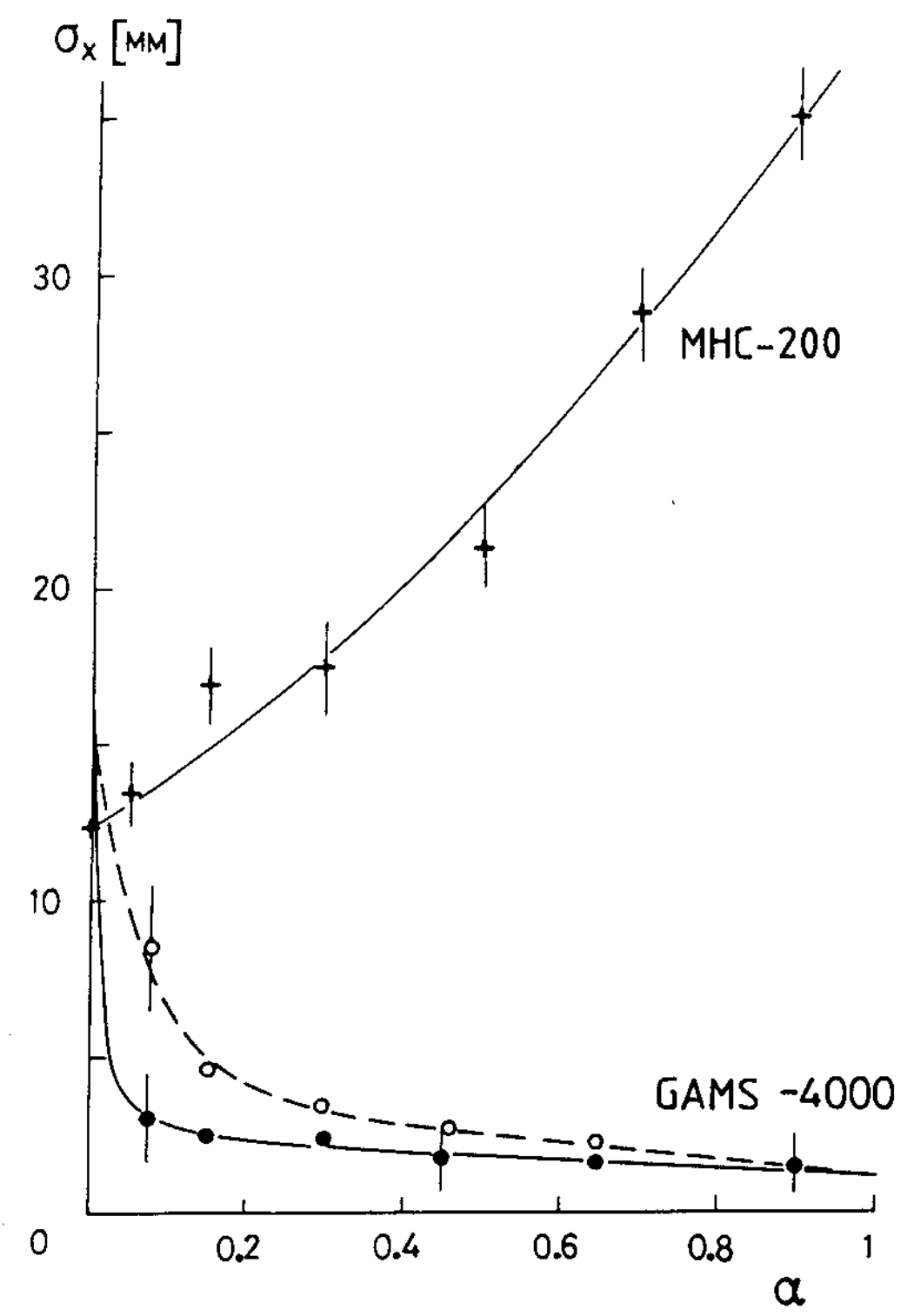

FIG. 7 

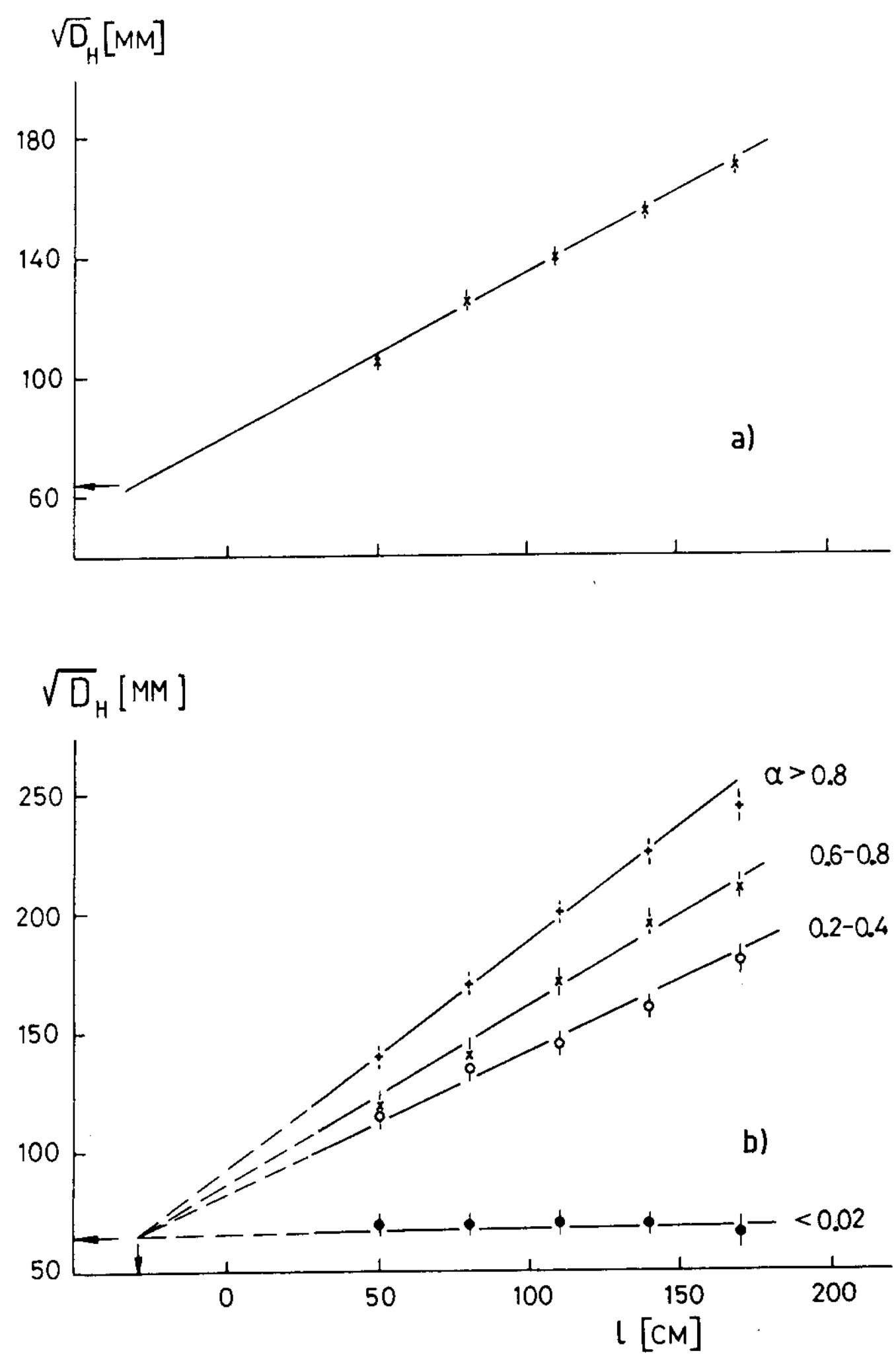

FIG. 8 

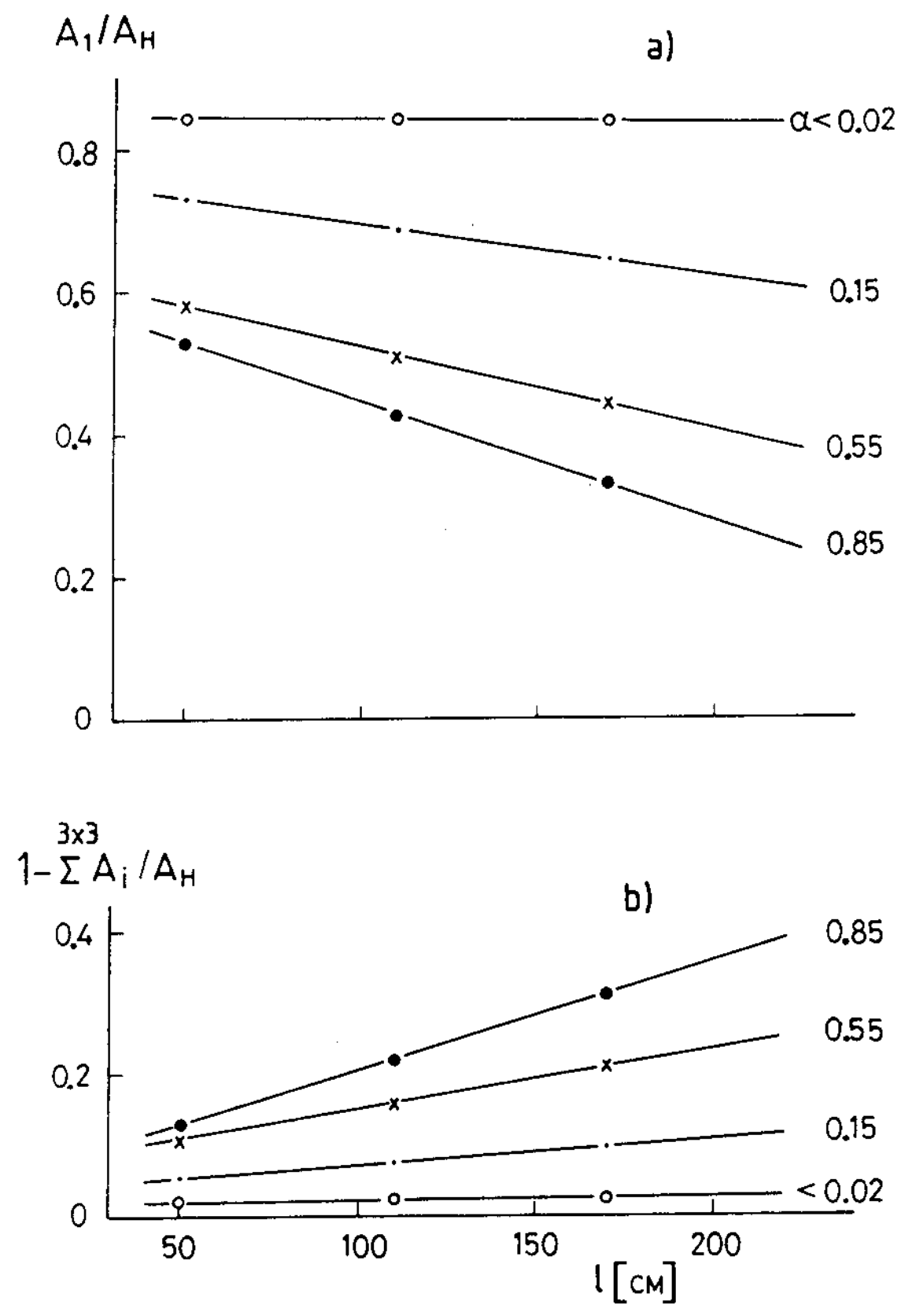

FIG. 9 

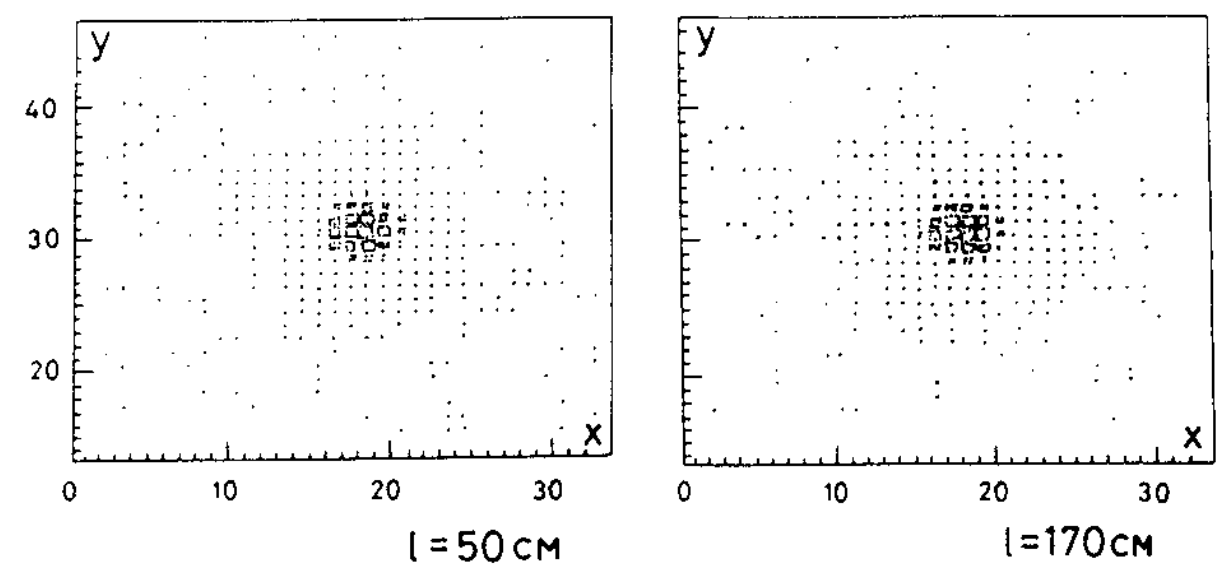

FIG. 10 